International Journal of Advanced Trends in Computer Science and Engineering

Available Online at http://www.warse.org/IJATCSE/static/pdf/file/ijatcse3881.12019.pdf

https://doi.org/10.30534/ijatcse/2019/3881.12019

\title{
Robot Advanced Intellectual Control developed through Flexible Intelligent Portable Platform
}

\author{
Luige Vladareanu, Victor Vladareanu ${ }^{1}$, Hongnian $\mathrm{Yu}^{2}$, Hongbo Wang ${ }^{3}$, Florentin Smarandache ${ }^{4}$ \\ ${ }^{1}$ Robotics and Mechatronics Dept. the Romanian Academy, Institute of Solid Mechanics, \\ Bucharest, 010141, Romania \\ luige.vladareanu@ vipro.edu.ro, victor.vladareanu@ vipro.edu.ro* \\ School of Computer Science and Network Security, \\ ${ }^{2}$ Dongguan University of Technology, Shongshanhu, Guangdong 523808, \\ China yu61150@ieee.org \\ ${ }^{3}$ Parallel Robot and Mechatronic System Laboratory of Hebei \\ Province, Yanshan University, Qinhuangdao, 066004, China \\ hongbo_w@ysu.edu.cn \\ Department of Mathematics, \\ ${ }^{4}$ University of New Mexico,705 Gurley Avenue, Gallup, NM 87301, USA \\ smarand@unm.edu
}

\begin{abstract}
This paper offers an intelligent, flexible, portable robot platform VIPRo, involving the development of intelligent control interfaces through the application of advanced control techniques which are modified to the robot environment. These include Robot Haptic Control (RHC), Robot Extenics Control (eHFPC), Robot Neutrosophic Control (RNC), human adaptive mechatronics, applied by high speed processing IT\&C techniques, and real time communications for processing large volumes of data. An original virtual projection methodology is used to SMOOTH firefighting robots by representing mobile intelligent robots in a threedimensional virtual environment using VIP-F ${ }^{2}$ Ro with a robotic strong simulator, an open architecture system, and flexible networks over the classic robot control system.
\end{abstract}

Key words: VIPRO platform, robot simulation, graphical user interface, reference generation.

\section{INTRODUCTION}

Mobile robots have caught the attention of the research community and the manufacturing industry as well, leading to a great hardware and software developing. Some applications of great interest for researchers are human behaviour in fires and the simulation of the movement of individuals in such hazardous environment [1-3]. Simultaneously, the real time robot control with remote network control having human operators' ability play an important part in hazardous and challenging environments of human life exposed to great dangers such as support and repair in nuclear contaminated area, fire, earthquake or any other disaster area in case of an accident or a terrorist attack involving CBRN materials. [2-3]. A big amount of researches led to the development of different robots with sensing abilities, transport and manipulation of different applications [4-7].
This calls further developing of the mobile and remote control self-ruling robots which can help individuals to perform seeking and sparing activities, speaking to a priority and a complex task.

Generally, the total impact cost of larger episodes is extremely high and is higher than that of the countermeasures. For a biological threat, indirect economic impacts are evaluated so they are in the range of billions, to tens of billions, of US dollars. The countermeasure cost range is lower, and ranges from hundreds of millions to approximately 10 billion USD. The bio-defense program is a few hundred thousand to tens of millions spent by European countries in a particular year, whilst the USA invests about 200 million Euros.

Intelligent heterogeneous robot networks, controlled remotely by humans, have a vital role in challenging and hazardous environments, where lives might be at risk [8-10]. This is a challenge for developing autonomous systems perceptive to human requirements which can continuously learn, adapt and improve in "real world" complex environments to give support in natural disasters, fires, etc. [14-17].

The paper offers a VIPRo versatile, intelligent robot platform, which develops intelligent control interfaces through the application of advanced control techniques which are adapted to the robot environment, such as Robot Haptic Control (RHC), Robot Extenics Control (eHFPC), Robot Neutrosophic Control (RNC),human adaptive mechatronics, etc. An original virtual projection methodology is applied to SMOOTH firefighting robots, via development of the VIP$\mathrm{F}^{2}$ Ro Platform, allowing a representation of intelligent mobile robots in a 3D virtual environment using a strong robotic simulator, an open architecture system and adaptive networks over the classical robot control system. 
The VIP-F² Ro Virtual Intelligent Portable platform, is the one designed to acquire the data received from unmanned ground vehicles (UGV), to process and analyse them, to provide feedback. The VIP-F² Ro brings the virtual robots to the real world, wanting to create an innovative robot platform, which will allow developing mechatronic systems of mobile robots in virtual environments and communicating with real robot systems through a high speed interface.

The obtained results lead to the conclusion that the advanced intelligent robot control methods using neutrosophic control, extended control (Extenics), human adaptive mechatronics, developed through versatile intelligent portable platform, allow a correct evaluation of robot behaviours in hazardous or challenging environments and improving the robot performances at the interaction with the environment.

\section{VERSATILE INTELLIGENT PORTABLE PLATFORM}

The VIP-F ${ }^{2}$ Ro Virtual Intelligent Portable Platform for firefighting robots has been developed by an e-learning and remote-control platform which enables communities interested in the topic and long-term plans for the additional development of innovation and research. This is the tool which ensures the ability to continuously learn, adapt and improve in "real world" complex environments, modeling in real time information collected by advanced technologies to provide support in "big data" management and international cluster development which can process the information in a unifying vision.

This way, networking activities will be in good balance with scientific and technical activities contributing equally to advance the project and to achieve the specific objectives mentioned above $[18,20]$.

For the development of new features for the unmanned ground robotic mobile vehicle, such as motion on uneven ground, or motion by bypassing or overcoming obstacles, high level intelligent algorithms need to be developed. This is because the motion mechanism is a complicated process, and because it is a repetitive process of tilting and unstable movements which can occur on a bumpy road, it will cause the robot to tip over.

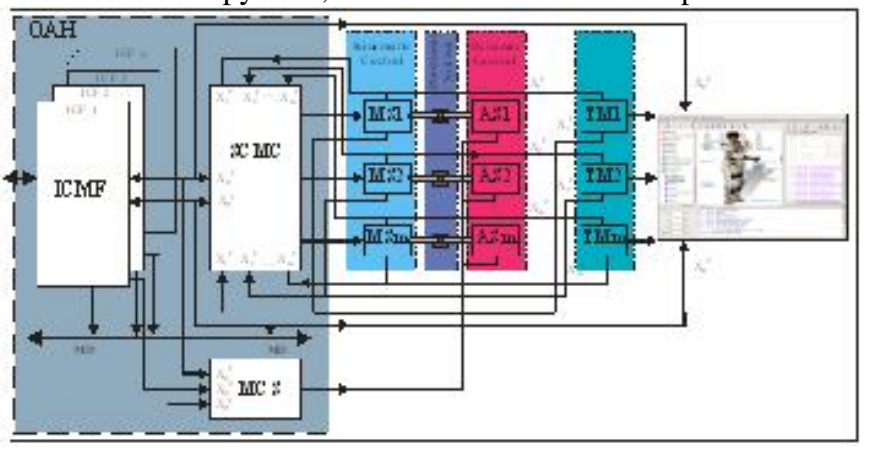

Figure: 1: Virtual projection method by VladareanuMunteanu applied to VIP-CBRN Platform
The virtual projection method [19-20] (Figure 1) is used to test the performance of dynamic position-force control through integrating dynamic control loops using a Bayesian interface for the neutrosophic interface and sensor network for decision making [9-11]. The CMC classical mechatronic control directly controls servomotors MS1, MSm, where $\mathrm{m}$ is the number of the robot's degrees of freedom. The signals are sent to a virtual control interface (VCI), this processes them and creates the necessary signals for $3 \mathrm{D}$ graphical representation on a graphical terminal CGD. Developing an open architecture control system by integrating $\boldsymbol{n}$ control functions as well as to those supplied by the CMC mechatronic control system. With this assistance new control methods can be introduced, such as: control of the center of gravity, motion control schemes, contour tracking functions, orientation control through image processing, decision making by neutrosophiclogic control, and Bayesian interface for sensor networks $[11,12]$. Real time control, priority control, and information exchange management between the $\mathrm{n}$ interfaces is controlled by the multifunctional control interface MCI, connected via a high speed databus.

The optimization of intelligent control methods allows the Unmanned Ground robotic mobile Vehicle (UGV) to adapt to environmental scene of in case of the fire investigation, hazardous chemicals detection, fire and rescue threat the firefighter's safety and life, through real time control, without losing its stability during the mission.

To model through adaptive mechatronic methods of the robot implemented by the intelligent, versatile, and portable robot, the VIPRO platform presents three intelligent control interfaces (ICs). Human adaptive mechatronics are intelligent mechanicalelectrical systems which can adapt to human skills in different environments and provide assistance to improve the skill and overall operation of the combined human machine system to improve performance. The VIPO platform architecture, in correlation with the virtual projection method (Figure 1) is developed in Figure 2

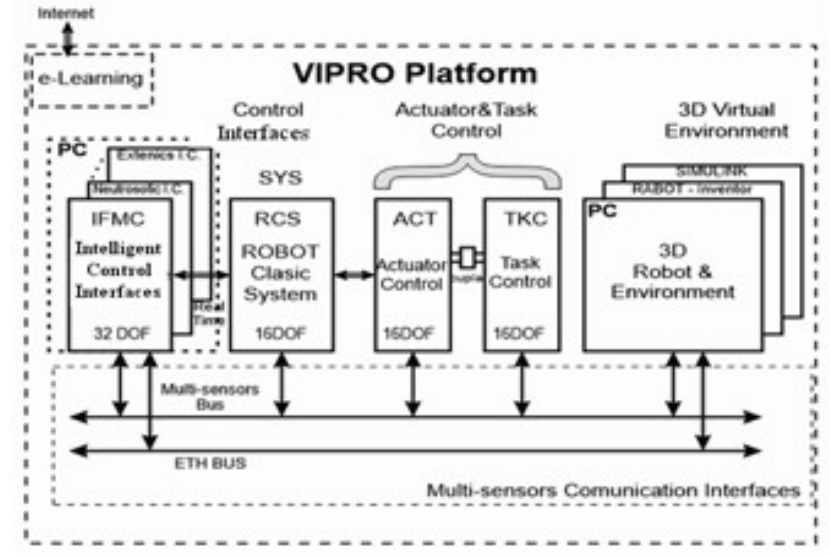

Figure: 2 Integration of the VIP-F ${ }^{2}$ Ro Platform in the VIPRO Platform architecture 
The results of simulation investigation and identifying the features and parameters of the virtual intelligent platform VIP$\mathrm{F}^{2} \mathrm{Ro}$ are obtaining by simulation studies. These will be used to establish the UGV optimal parameters for intelligent interfaces development. VIP Platform allows intensive simulation studies for damping motion, motion compensation, UGV swing amplitude, UGV rotation/advance, motion timing, motion orientation, UGV tilt over, landing position.

The VIP-F ${ }^{2}$ Ro platform technical solution contains the intelligence control interface module, which utilizes advanced control strategies which are adapted to the robot environment, such as extended control - extenics, neutrosophic control human adaptive mechatronics, etc., used via various IT\&C techniques, with real time communication and fast processing. This module mainly contains the interface for intelligent neutrosophic control through integration of the RNC (Robot Neutrosophic Control) method [12], known as VladareanuSmarandache method, Extended Control Interface through Extenics (ICEx) [10, 13] and Haptic Robot Control Interface (CRH) [9-11].
The control system is comprised of proposed intelligent control interfaces: neutrosophic control interface (ICN) integrating neutrosophic robot control (RNC), extended control interface (ICEx) which is integrated through an extended hybrid force- position control (eHFPC) and the multifunctional control interface (ICM). Additionally, the haptic robot control interface (CRH) is used for movement and navigation in uncertain environments and on uneven terrain.

\section{ADVANCED INTELLIGENT CONTROL OF THE SMOOTH ROBOT, THROURGH VIP PLATFORM}

The new virtual intelligent portable platform of firefighting robots, VIP- $\mathrm{F}^{2} \mathrm{Ro}$, is the one designed to acquire the data received from unmanned robotic vehicles, to process and analyse them, to provide feedback. The , VIP- $\mathrm{F}^{2}$ Ro brings the virtual robots to the real world, wanting to create aninnovative robot platform, which will allow to develop mechatronic systems of mobile robots in virtual environments and communicate with real robot systems through a high speed interface

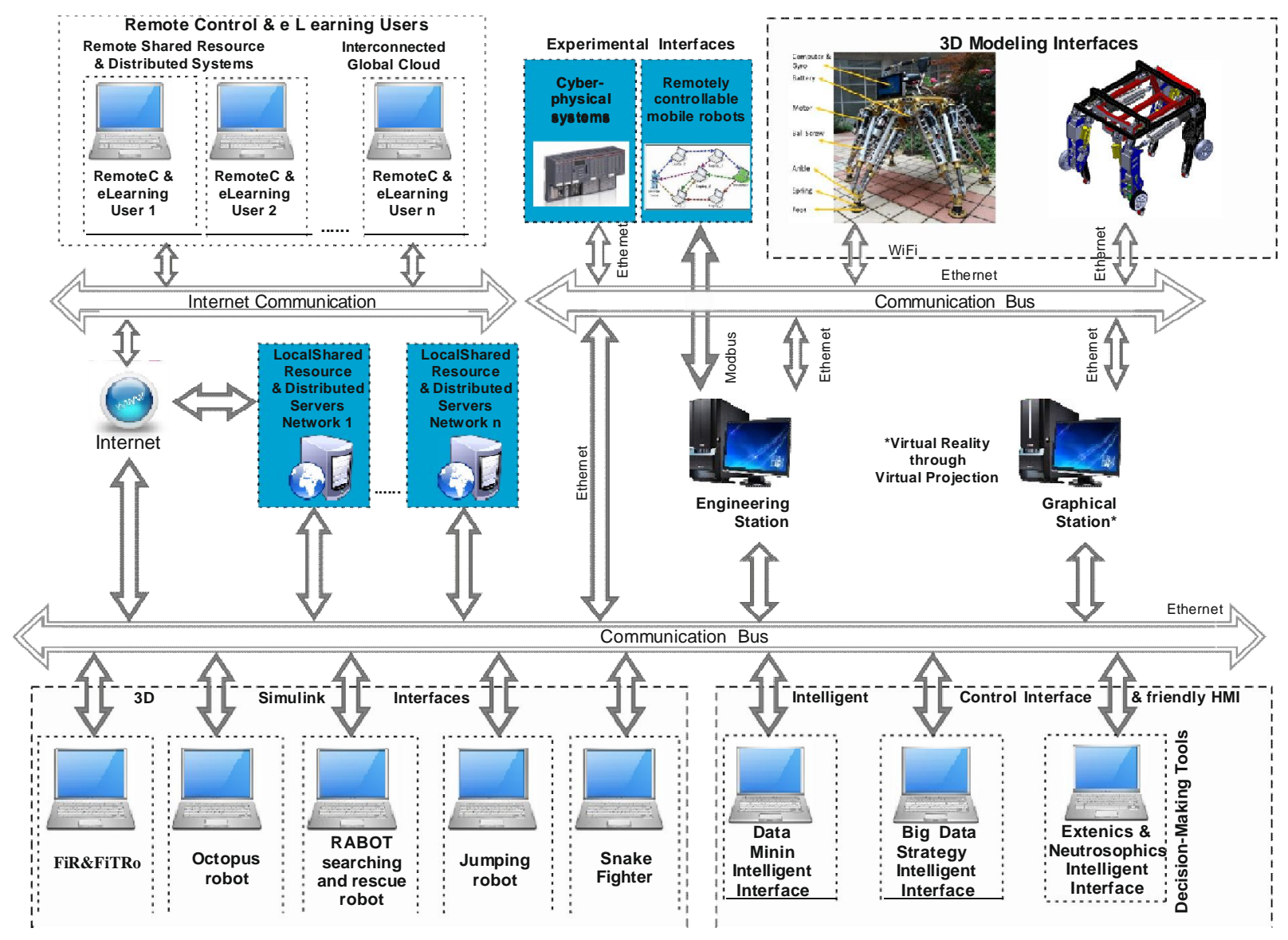

Figure 3: The VIP- $\mathrm{F}^{2} \mathrm{Ro}$ - Virtual Intelligent Portable Platform of the SMOOTH firefighting robot

The predictions and outputs from the generated models may be used innumerous ways Often, in circumstances such as fire growth, the results may be directly sent to personnel at the fire ground or to other community services. If the model predicted that the fire may spread into a section of the building where toxic compounds are stored, the model may integrate 
with a smoke- generation model and weather model for predicting any potential impact on surrounding communities.

The information is then directly sent to law enforcement agencies, disaster management departments, and local hospital stone able potential evacuation planning and treatment of victims. Usually, predictions and model outputs would drive real-time 3D visualization of the fire ground, personnel, and equipment. The ICs then display the evolution of the fire incident to analyze the possible impact of actions and decisions before issuing commands to personnel. The visualization is then recorded for future analysis, training, and lessons learned.

The computational platform VIP-F ${ }^{2}$ Ro designed in this project will be based on the virtual projection method. VIP$\mathrm{F}^{2}$ Ro is extendable for integration, testing and experimenting of firefighting environments through building an open architecture system and adaptive networks, combining the expertise of a team of specialists in fire engineering, electronics, mathematics, computer sciences with the expertise of a diverse group of researchers in different fire specialties.

The innovative platform VIP- $\mathrm{F}^{2}$ Ro (Figure 3), has been developed as an open architecture system and adaptive network which integrates Future Internet Systems vision enabling: intelligent network control systems, cyber-physical systems by adaptive networks, big data, data mining, human in the loop principles, network quality of service, intelligent control interfaces, shared resources, and distributed server network - remote control and e-learning users by interconnected global clouds. Thus, the challenges and expected progress of VIP- $\mathrm{F}^{2}$ Ro are its ability to be competitive, interactive, and integrated with advanced scientific research concepts.

The idea is that the robotics mobile unit will go to the safe proximity of the firefighting emergency area, in particular fire and rescue operations such as aircraft/airport rescue, wilderness fire suppression, and search and rescue, including emergency medical services. It can do that as it is equipped with innovative devices that determine the direction and the identification of dangerous clouds and the toxic environment created by combustible materials, their moving direction, nature of agents that contaminate, oxygen deficiency, elevated temperatures, and poisonous atmospheres, provided in safe condition for personnel protection. After the safe stop of robotics mobile unit, there are the correlated actions of unmanned ground and aerial vehicles (VIP- F $^{2}$ Ro and UAV), all of these coordinated by the virtual intelligent platform, as follows next.

The requirement for managing every interaction and behavior is solved through the development of a new interface for intelligent control based on advanced control strategies, such as extended control (Extenics), human adaptive mechatronics, neutrosophic control, implemented by high speed processing IT\&C techniques in real time communication for a high volumes of data processing, including a remote control \& e-learning component and an adaptive
This permits the development of new methodologies, test platforms, evaluation metrics, experiment reproducibility, novel approaches to academia-industry co-operation, of the products and process innovation and a fire engineering network for modeling and researching complex data for firefighting quick actions, and management of fire and emergency services.

Robotic control is fundamental in the development of control and perception algorithms for robotics applications. A 3D simulator for mobile robots needs to be able to correctly control the robot dynamics and objects in the environment. Additionally, real- time control is vital to correctly model interactions amongst and between robots and the environment, therefore it is frequently necessarily to approximate to obtain real-time performance.

The innovative firefighting robotic mobile ground vehicle, is sent for support to people, physical evaluation, examination and collection of material / evidence. Some "plus (+)" aspects of this innovative firefighting robot are: high stability and ease of remote control (manoeuvrability) in severe ground topography and / or narrow spaces like pipes; modular structure with, relatively, low costs specific components; ability to work in natural disasters and emergency incidents threatening life and property.

The networked real- time control will be distributed and decentralized using multi-processor devices for fusion control, data reception from transducers mounted on the robot, peripheral devices connected through a wireless LAN for offline communications and CAN, MODBUS, PROFIBUS or ETHERNET fast communication network for real time control. The VIP- $\mathrm{F}^{2}$ Ro system was designed in a distributed and decentralized structure to enable development of new applications easily and to add new modules for new hardware or software control functions. Moreover, the short time execution will ensure a faster feedback, allowing other programs to be performed in real time as well, like the apprehension force control, objects recognition, making it possible that the control system have a human flexible and friendly interface.

The VIP-F ${ }^{2}$ Ro Platform develop the intelligent interfaces using Robot Neutrosophic Control (RNC), Robot Extenics Control Interface (eHFPC) and Robot Haptic Control (RHC) Interface for Unmanned Ground robotic mobile Vehicle (UGV) which acts in correlation and interaction with Unmanned Aerial Vehicle (UAV) through implementation of the network mobile robot system over Mobile Ad-hoc Network. The target robot is equipped with a robotic arm to execute various tasks. The relay / observer robot can route network packets between the controller and the target robot. It also produces visual feedback of the target robot to the user at the controlling end. 


\section{HAPTIC INTELLIGENT CONTROL INTERFACES}

Recently haptic interfaces have become a reliable solution for solving problems which arise when humans interact with the environment. In research areas of haptic interaction between humans and the environment there is important research, an innovative approach for interactions between the robot and the environment using haptic interfaces and virtual projection method is present adhere. To control this interaction, we used the Virtual Projection Method where haptic control interfaces of impedance and admittance will be embedded.

For moving of the firefighting robots in uncertain environments, allowing actuation in crisis situations or natural disaster, in which human life is in danger, SMOOTH will develop haptic interfaces that provides the robot spatial orientation and navigation based on that the robot feels the land on which it moves by changing the stiffness of the robot paw joints and of the segments robot joints, using the stiffness associated of the paw joints position $\mathrm{X}_{\mathrm{C}}$ on the robot environment map if uneven ground is detected [9-11].

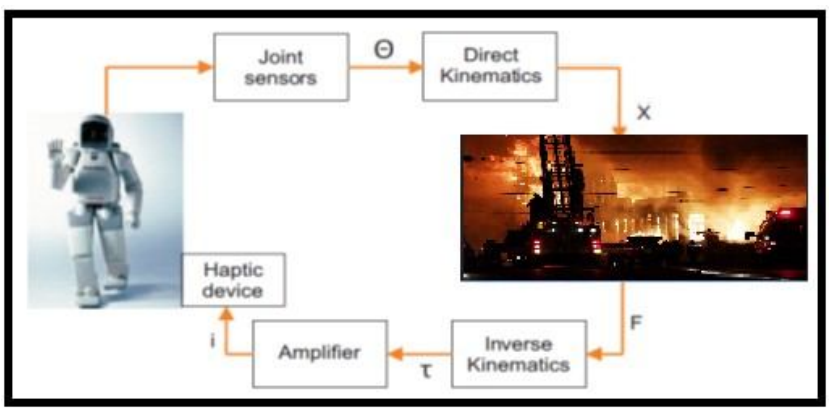

Figure 4: Haptic interfaces for firefighting robots using VIP-F ${ }^{2}$ Ro Platform

This leads to successively change the robot movement scheme and change the position control loop to the force control. Thus, the human operator can remotely control the robot's movement through two parameters, first visual and the second haptic (Figure 4). Respectively, the human operator sees the robot environment map and simultaneously feels damping of the robot leg movement at actuation of the haptic device lever, with the possibility of generating the haptic Cartesian positions $\mathrm{X}_{\mathrm{CH}}$ for adapting the robot movement, on uneven and unstructured terrain.

Haptic interfaces are intended to reproduce or include the sense of touch through manipulation or, perception of real environments using computer control and mechatronic devices. They consist of a haptic device and a computer, which incorporates software that associates input data with haptic information rendering. Figure 4 shows how haptic interfaces work and the ways in which it will be implemented for controlling fire fighting robots using the VIP-F ${ }^{2}$ Ro Platform.

The innovative solution developed and patented for haptic robot control allows the robot to "feel" the terrain on which the mobile autonomous robot moves by the modification in rigidity of the joints and of the joints segment when detecting unevenness depending on the rigidity $\mathrm{K}_{\mathrm{Xc}}$ associated to the joint position of the robot $\mathrm{X}_{\mathrm{C}}$ on the robot environment map. Modifications in rigidity are realized from the time the joint touches the terrain until complete contact of the joint segment. The human operator has the possibility to remotely control the robot movement, through two parameters, one visual and the second haptic, respectively seeing the robot environment map and simultaneously to feel remotely the dampening of the robot joint movement when using the haptic device stick. Depending on the type of manipulation of the haptic device, the human operator generates the haptic Cartesian positions $\mathrm{X}_{\mathrm{CH}}$ to ensure the robot motion is adapted to the uneven and unstructured terrain in crisis situations or natural disasters where human lives may be at risk.

To generate the robot environment map, a CCD camera is used to process the images, stabilized for various robot motion directions. This is achieved by processing the signals received from a 3D gravitational transducer (TGR3D) and a magnetic compass (TBM), which results in an interface of the 3D robot environment map with a stable image to the robot movement. Each of the points in the robot environment map areassociated with the rigidity of the robot joint position $\mathrm{X}_{\mathrm{C}}$, named the associated rigidity $\mathrm{K}_{\mathrm{Xc}}$. Movement damping at contact between the robot joint and uneven terrain is obtained by switching from the position control to the force control when the tip or joint posterior touches the terrain, depending on the robot motion scheme, until total contact of the joint segment is achieved.

Haptic control of the robot movement by the human operator occurs via a haptic device permitting the human operator to feel the robot joint movement damping and generates the Cartesian reference positions of the robot movement, called haptic Cartesian positions $\mathrm{X}_{\mathrm{CH}}^{\mathrm{H}}$, for adaptation of robot movement to an unstructured and uneven terrain. The telemetry module (TL) permits measurement of the distance to the joint segment through the use of anoptical scanning device.

The novelty VIP-F ${ }^{2}$ Ro Virtual Intelligent Portable platform for firefighting robots, is competitive with other similar virtual simulation platforms with applications in robotics, called virtual instrumentation, CDA, CAM, CAE, Solid Works, etc., very powerful in modeling but only in a virtual environment, or the MatLab, Simulink, COMSOL, Lab View platforms, which allow extensions for real time data acquisition and signal processing. In addition to these, VIP-F ${ }^{2}$ Ro allow the experimental validation of intelligent control methods by integrating the classical robot real time control system in modelling, design, simulation and testing of the robot motion and stability.

\section{CONCLUSION}

Development of 3D dynamic perception and visualization, and human-robot interaction software systems are formidably challenging and accordingly the activities to support software 
developments and project management processes are of vital importance to this piece of research. Attribute selected techniques can be categorised on the basis of a number of criteria. Dynamic data come from environmental and wearable sensors, mobile robots and radio communications. SMOOTH will therefore develop software systems for real-time data analytics to assess situational awareness, asses risk and improve decision-making by firefighters and ICs. New computational software tools and virtual reality engines are being developed to support both risk and the decisions. The VIP-F²Ro Platform also develop adequate metrics and testing tools to determine the effectiveness and validity.

This is part of a larger effort to completely define a virtual environment for the simulation and testing of mechatronic systems on a remote virtual platform, encompassing all the usual and innovative aspects in the field of Robotics research, from low-level actuator control and mechanism design to intelligent operational strategies and environment configuration modelling. It has the advantage of allowing virtually all manner of testing to be made remotely, with little or no extra configuration cost, while reducing the risk of equipment damage and maintaining the realism and end-result application value that can onlycome with actual hardware testing. This approach combines the best features of both scientific lines of enquiry, software simulation and direct hardware implementation.

Major outcome of this work is development of an Integrated Safe Smart Robotics Mobile Unit \& Virtual Intelligent Platform for Remotely - Controlled Technologies in the fire investigation, hazardous chemicals detection, fire and rescue threat the firefighter's safety and life in emergency situations. Its innovation potential comes from the fact that it integrates, through VIP-F² Ro Platform, both UGV (with innovative robotic arm module) and UAV (with innovative sensors and miniature sensors). This is how it enables intervention in various ground condition (uneven terrain, narrow spaces) where examination by humans may not be possible, or could be severely restricted. It allows searching and rescuing in smart firefighting control, safe operating in highly contaminated radioactive and chemical environments, and to facilitate the decision making with higher efficiency and collecting evidence / data which are further automated processed and generated reports are transmitted to decision centre. Also, prediction and local prognoses on highly contaminated areas are available.

\section{ACKNOWLEDGMENT}

This work was developed with the support of MENUEFISCDI, PN-II-PT-PCCA-2013-4, VIPRO project No.009/2014-2017, Romanian Academy, "Joint Laboratory of Intelligent Rehabilitation Robot" collaborative research agreement between Romanian Academy by IMSAR, RO and Yanshan University, CN, project KY201501009/2016-2018 and H2020-MSCA-RISE-2016/H2020-MSCA-RISE-2016, SMOOTH project, GA no. 734875 , 2016-2020.

\section{REFERENCES}

[1] Murphy, Robin R. "Human-robot interaction in rescue robotics." IEEE Transactions on Systems, Man, and Cybernetics, Part C (Applications and Reviews) 34.2 (2004): 138-153. https://doi.org/10.1109/TSMCC.2004.826267

[2] Hongnian $\mathrm{Yu}(\mathrm{UK})$, L. Vladareanu (RO), H. Wang (CN), Z.G. Hou $(\mathrm{CN})$,

F. Gao (CN), FP7-PEOPLE-2012-IRSES RABOT project no. 318902, 2012-2016
[3] Hongnian Yu (UK), L. Vladareanu (RO), H. Wang (CN), Z.G. Hou $(\mathrm{CN})$,

F. Gao (CN), V. Somoghi (RO), R. Lopez (SP), F. Claeyssen (FR), H2020-MSCA-RISE-2016/H2020MSCA-RISE-2016, SMOOTH project, GA no. 734875 , 2016-2020

[4] Yang Liu, Hongnian Yu, Luige Vladareanu, Shuang Cang, and T. C. Yang, "Trajectory Optimization of a Pendulum-Driven Underactuated Cart", SISOM 2011 and Session of the Commission of Acoustics, pp 287- 299, Bucharest 25-26 May, 2011

[5] O. Formsma, N. Dijkshoorn, S. van Noort and A. Visser (2011), "Realistic Simulation of Laser Range Finder Behavior in a Smoky Environment", in "RoboCup 2010: Robot Soccer World Cup XIV", Lecture Notes on Artificial Intelligence series, volume 6556, p. 336-349, Springer, Heidelberg, March 2011.

https://doi.org/10.1007/978-3-642-20217-9_29

[6] Vladareanu L., A.Curaj, R.I.Munteanu, Complex Walking Robot Kinematics Analysis And PLC Multi-Tasking Control, Revue Roumaine des Sciences Techniques Série Électrotechnique et Énergétique, 2011, Volume: 57 Issue: 1 Pages: 90-99 Published: JAN-MAR 2012, WOS:000303096800010, ISSN 0035-4066.

[7] Yi Dong, Hongnian Yu, Feng Gao, Design, Modelling and control of a light touch Mechanism, International Conference on Advanced Mechatronic Systems, Zhengzhou, China, 11-13 August 2011.

[8] R. R. Murphy, "Human-Robot Interaction in Rescue Robotics", IEEE Trans on Systems, Man, Cybernetics, Part C, vol.34, no. 2, pp.138-158.

[9] Victor Vladareanua, Radu I. Munteanub, Ali Mumtazc, Florentin Smarandached and Luige Vladareanua, "The optimization of intelligent control interfaces using Versatile Intelligent Portable Robot Platform", Procedia Computer Science 65 (2015): 225 - 232, ELSEVIER, www.sciencedirect.com, doi:10.1016/j.procs.2015.09.115. https://doi.org/10.1016/j.procs.2015.09.115

[10] Luige Vladareanu, et. al, "Versatile Intelligent Portable Robot Platform using Adaptive Networked Control Systems of Rescue Robots", VIPRO Platform, project no. 009/2014, PN-II-PT-PCCA-2013-4, MENUEFISCDI, RO, 2014-2017

[11] Victor Vladareanu, Ovidiu Ilie Sandru, Luige Vladareanu, Hongnian Yu, "Extension Dynamical Stability Control Strategy for the Walking Robots", International Journal of Technology Management, SKIMA 2012, Inderscience Publisher, 2013, ISSN online: 1741-5276, ISSN print: 0267-5730, http://www.inderscience.com/jhome.php?jcode=ijtm

[12] Smarandache F., Vladareanu L., "Applications of Neutrosophic Logic to Robotics - An Introduction", The 2011 IEEE International Conference on Granular Computing Kaohsiung, Taiwan, Nov. 8-10, 2011, pp. 607-612, ISBN 978-1-4577-0370-6 https://doi.org/10.1109/GRC.2011.6122666

[13] Şandru Ovidiu Ilie, LuigeVladareanu, Paul Şchiopu, Victor Vlădareanu, Alexandra Şandru, "Multidimensional Extenics Theory", U.P.B. Sci. Bull., Series A, Vol. 75, Iss. 1, 2013, pg.3-12, ISSN 1223 7027

[14] Marina Shitikova, Luige Vladareanu, Claudio Guarnaccia, „Recent advances in mechanical engineering”, WSEAS Press, 2014, pg.207-2014, ISBN: 978-960-474-402-2 
[15] S.B. Balakirsky and Z. Kootbally, "USARSim/ROS: A Combined Framework for Robotic Control and Simulation", Proceedings of the ASME 2012 International Symposium on Flexible Automation (ISFA 2012), St. Louis, June 18-20, 2012 https://doi.org/10.1115/ISFA2012-7179

[16]Cononovici, Sergiu Boris, and Adrian Curaj. "An approach to walking robots planning and control." Proceedings of the Romanian Academy (2010): 75-82.

[17] Y.F. Feng, H.B. Wang, T.T. Lu, et al. "Teaching training method of a lower limb rehabilitation robot." Int J Adv Robot Syst, vol. 13, pp. 1-11, February 2016. https://doi.org/10.5772/62058

[18] Cononovici, S.B., Vlădăreanu, V., et al. "Control Strategies for Synchronous Generation of Resistive Loads Correlated to the Robot Motion Environment", $5^{\text {th }}$ International Workshop on Cyber Physical Systems (IWoCPS-2016), Romanian Academy, Bucharest.

[19] Vladareanu Luige, et all, Method and device for the realtime control of robots by virtual, projection, EPO: A 200800232.

[20] Yang Liu, Hongnian Yu and Luige Vladareanu,An Iterative Learning Control Scheme for the Capsubot, UKACC International Conference on Control 2008, University of Manchester, UK, 2-4 September 2008. 